\title{
PWE-034 DIETARY ARACHIDONIC ACID AND THE AETIOLOGY OF ULCERATIVE COLITIS: DATA FROM A UK PROSPECTIVE COHORT STUDY, USING 7-DAY FOOD DIARIES
}

doi:10.1136/gut.2011.239301.297

P De Silva, ${ }^{1, *}$ R Luben, ${ }^{2}$ A Mc Taggart, ${ }^{3} \mathrm{~K}$ T Khaw, ${ }^{3}$ A Hart ${ }^{4}{ }^{1}$ Gastroenterology, The Ipswich Hospital NHS Trust, Ipswich, UK; ${ }^{2}$ Public Health and Primary Care, School of Clinical Medicine, University of Cambridge, Cambridge, UK; ${ }^{3}$ Clinical Gerontology Unit, School of Clinical Medicine, University of Cambridge, Cambridge, UK; ${ }^{4}$ School of Medicine, Health Policy and Practice, University of East Anglia, Norwich, UK

Introduction There is emerging evidence that arachidonic acid (AA), an n- 6 polyunsaturated fatty acid ( $\mathrm{n}-6$ PUFA), is involved in the aetiology of ulcerative colitis. The biological mechanism may involve the production from AA, of the proinflammatory eicosonoids prostaglandin $\mathrm{E}_{2}\left(\mathrm{PGE}_{2}\right)$ and leukotriene $\mathrm{B}_{4}\left(\mathrm{LTB}_{4}\right)$, which are present in higher concentrations in the colonic mucosa of patients. The aim of this study was to investigate, for the first time in a prospective cohort study using nutrient data from 7-day food diaries (7-DFDs), whether an increased dietary AA intake was associated with the development of incident ulcerative colitis.

Methods A total of 23658 men and women aged 40-78 years, in Norfolk, UK, were recruited into EPIC (European Prospective Investigation Into Cancer). At baseline, participants completed detailed 7-DFDs which recorded their dietary intake of food types, brands, quantities, frequency of consumption and cooking methods. Each diary was coded by nutritionists, using a specifically designed computer programme which contained information on the nutrient content of 11000 food items, including AA. The cohort was then monitored for participants who subsequently developed incident ulcerative colitis, with each case confirmed by review of the medical notes. Each case was matched for age at recruitment, date of recruitment, gender and follow-up time, with four controls, and the odds ratios calculated for the risk of ulcerative colitis and tertiles of AA intake. The analysis was adjusted for cigarette smoking, total energy intake and other dietary fatty acids affecting AA metabolism.

Results In the cohort, 26 participants (58\% men) developed incident ulcerative colitis after a median follow-up time of 3.8 years (range $0.5-8.3$ years). The extent of the colonic inflammation was up to the splenic flexure in $53 \%$ of patients. Higher AA intakes were associated with an increased risk of UC (highest vs lowest tertile $\mathrm{OR}=6.09,95 \% \mathrm{CI}=1.05$ to $35.23, \mathrm{p}=0.04$ ) with a positive trend across tertiles $(\mathrm{OR}=1.94,95 \% \mathrm{CI}=0.88$ to $4.29, p=0.10$ ). The attributable fraction for the two higher tertiles of AA intake and incident cases of UC was $57 \%$.

Conclusion An increased dietary intake of arachidonic acid, as measured from 7-DFDs (the most accurate feasible method of measuring diet in large epidemiological studies), was associated with an increased risk of developing UC. While it is possible that preclinical disease might influence, dietary intake, these results further support a role for AA and $n-6$ PUFAs in the pathogenesis of UC, and their intake should be measured in future aetiological work.

Competing interests None.

Keywords arachidonic acid, ulcerative colitis. 